\title{
Explicit behavioral detection of visual changes develops without their implicit neurophysiological detectability
}

\author{
Pessi Lyyra $^{1 *}$, Jan Wikgren ${ }^{1}$, Timo Ruusuvirta ${ }^{2}$ and Piia Astikainen ${ }^{1}$ \\ 1 Department of Psychology, University of Jyväskylä, Jyväskylä, Finland \\ 2 Turku Institute for Advanced Studies, Department of Psychology, University of Turku, Turku, Finland
}

\author{
Edited by: \\ Hans-Jochen Heinze, University of \\ Magdeburg, Germany \\ Reviewed by: \\ Juliana Yordanova, Institute of \\ Neurobiology, Bulgaria \\ Ronald Rensink, University of British \\ Columbia, Canada \\ ${ }^{*}$ Correspondence: \\ Pessi Lyyra, Department of \\ Psychology, University of Jyväskylä, \\ PO Box 35, Fl-40014, Jyväskylä, \\ Finland. \\ e-mail: pessi.lyyra@jyu.fi
}

Change blindness is a failure of reporting major changes across consecutive images if separated, e.g., by a brief blank interval. Successful change detection across interrupts requires focal attention to the changes. However, findings of implicit detection of visual changes during change blindness have raised the question of whether the implicit mode is necessary for development of the explicit mode. To this end, we recorded the visual mismatch negativity (VMMN) of the event-related potentials (ERPs) of the brain, an index of implicit pre-attentive visual change detection, in adult humans performing an oddball-variant of change blindness flicker task. Images of $500 \mathrm{~ms}$ in duration were presented repeatedly in continuous sequences, alternating with a blank interval (either $100 \mathrm{~ms}$ or $500 \mathrm{~ms}$ in duration throughout a stimulus sequence). Occasionally $(P=0.2)$, a change (referring to color changes, omissions, or additions of objects or their parts in the image) was present. The participants attempted to explicitly (via voluntary button press) detect the occasional change. With both interval durations, it took 10-15 change presentations in average for the participants to eventually detect the changes explicitly in a sequence, the $500 \mathrm{~ms}$ interval only requiring a slightly longer exposure to the series than the $100 \mathrm{~ms}$ one. Nevertheless, prior to this point of explicit detectability, the implicit detection of the changes $\mathrm{VMMN}$ could only be observed with the $100 \mathrm{~ms}$ intervals. These findings of explicit change detection developing with and without implicit change detection may suggest that the two modes of change detection recruit independent neural mechanisms.

Keywords: change blindness, flicker paradigm, oddball paradigm, event-related potentials, visual mismatch negativity

\section{INTRODUCTION}

The human visual system is equipped with an automatic mechanism for detecting sudden changes in the environment. Abrupt stimulus appearance or motion captures attention in a bottomup way, even if these are not targets of visual search (Yantis and Jonides, 1984). However, this mechanism is prone to error if the load of attention is increased (inattentional blindness, see Mack and Rock, 1998) or if the change occurs simultaneously with some interruptive events. Massive changes may go easily unnoticed by this way, a phenomenon usually known as "change blindness" (for reviews, see Simons and Levin, 1997; Rensink, 2002). Change blindness can be observed as a failure to detect a considerable and otherwise easily detectable change when a flash of light, blink, saccade, or a large transient stimulus immediately precedes the change. The flicker paradigm, where a brief blank screen (or interstimulus interval, ISI) separates the two images across which a change is introduced, is a method that has firmly established the phenomenon of change blindness. It is also the most frequently used paradigm to induce the effect experimentally (Rensink et al., 1997).

Change blindness, as observed through failures in tasks to voluntarily (explicitly) report on the occurrences of the changes, is held to entirely reflect errors in top-down guided focal attention (Rensink et al., 1997; O'Regan and Noë, 2001). However, there is also accumulating behavioral (Hayhoe et al., 1998; Fernandez-Duque and Thornton, 2000; Hollingworth et al., 2001; Fernandez-Duque et al., 2003; Koivisto and Revonsuo, 2003; Laloyaux et al., 2006) and brain imaging (Beck et al., 2001; Huettel et al., 2001; Niedeggen et al., 2001; FernandezDuque et al., 2003; Pessoa and Ungerleider, 2004; Eimer and Mazza, 2005; Schankin and Wascher, 2007, 2008; Kimura et al., 2008; Khittl et al., 2009; Lyyra et al., 2010) evidence of implicit change detection by the visual system during change blindness. It has been suggested that changes are initially detected implicitly to guide focal attention to the locus of the changes and, thereby, to eventually allow the explicit detection and identification of changes supported by focal attention (cf. Chun and Nakayama, 2000; Niedeggen et al., 2001). Similarly, there is both behavioral (Watanabe, 2003) and electrophysiological evidence that changes are implicitly localized before their identification already during change blindness (Schankin and Wascher, 2007, 2008) or "sensing" the presence of change without identification (Busch et al., 2010a,b) suggesting that change localization and identification follow each other sequentially in the same visual processing stream. This view regards implicit change detection as a pre-requisite for explicit change detection. However, the 
dissociation between neural mechanisms for the (explicit) identification and (implicit) localization of a visual object (Goodale and Milner, 1992) also leaves open a possibility that explicit and implicit modes of visual change detection may recruit distinct and mutually independent neural mechanisms. This possibility is also indicated by distinct ERP-markers found for explicit and implicit change detection Fernandez-Duque et al., 2003; Kimura et al., 2008. Furthermore, while explicit change detection is associated with attentional visual short-term memory with a limited capacity (e.g., Rensink, 2000), it is possible that the neural traces of implicit change detection is held to be solely supported by visual sensory memory with limited time span and reportability of its contents (Sperling, 1960). The observation of distinct markers cannot, however, yield direct evidence in support of independent functioning between explicit behavioral change detection and implicit change detection. To substantiate this possibility, it should be shown that explicit change detection could occur with and without the presence of implicit change detection.

To this end, we investigated whether explicit detection of visual changes, as indexed by voluntary behavioral responses to them, can only develop when implicit detection of these changes, as reflected by the visual mismatch negativity (vMMN) of eventrelated potentials (ERPs) of the brain (for a review, see PazoAlvarez et al., 2003; Czigler, 2007; Kimura et al., 2011), also takes place. vMMN is a neurophysiological index of pre-attentive detection of visual changes on the basis of visual sensory memory (e.g., Astikainen et al., 2008). Its pre-attentive nature is reflected by the fact that, unlike with explicit change detection, voluntary attention need not be directed toward the changes to detect them. Its implicit nature is, in turn, reflected by no need to be declaratively aware of the changes. vMMN is elicited at the posterior electrode sites at around 150-300 ms following change (e.g., Czigler, 2007). Although explicit detection of visual changes is relatively insensitive to the duration of the blank interval separating the changed and unchanged images (Rensink et al., 2000), vMMN, in contrast, is known to diminish when ISI is prolonged (Astikainen et al., 2008), reflecting most probably the time span of visual sensory memory (Sperling, 1960). A behavioral index of explicit detection of visual changes and $\mathrm{vMMN}$ as an index of their implicit detection is, therefore, a perfect combination to investigate whether explicit detection could develop without implicit one. Previously, in change blindness studies using short intervals or change-occluding stimuli between images $(30-100 \mathrm{~ms}$, Schankin and Wascher, 2007, 2008) change-related electrophysiological responses have been observed, but not in similar studies using longer intervals (500 ms: Eimer and Mazza, 2005; 900 ms: Henderson and Orbach, 2006). We thus tested the implicit detection of the changes as indexed by vMMN to an image with change as interspersed with a repeated image with no change. To vary the elicitability of vMMN, two durations of blank intervals (ISIs) between the images delivering the change were used: a short interval (100 ms ISI) expected to lead to vMMN, and a long interval (500 ms) unlikely to produce vMMN. ERPs were recorded in trials prior to the first explicitly detectable image with change. We hypothesized that if the explicit detection of the changes is conditional upon their implicit detection, the explicit detection of the changes should fail if vMMN fails to be generated by images with change (with longer ISIs). If, on the other hand, the explicit detection of the changes can bypass visual sensory memory and directly recruit attentional memory (Rensink et al., 1997), the explicit detection should emerge even when vMMN remains unobservable in preceding trials.

\section{MATERIALS AND METHODS PARTICIPANTS}

Twenty-two volunteers, 14 female and eight male with age distribution of 19-33 years (mean age 23.2 years, s.e. 4.03 ) participated in the study. All of them had normal or corrected-to-normal vision. Before the experimental treatment, the participants were informed about the nature and purpose of the study, and a written consent was obtained from them approved by the ethical committee of University of Jyväskylä. The study conforms to The Code of Ethics of the World Medical Association (Declaration of Helsinki).

\section{STIMULI AND PROCEDURE}

Participants viewed the stimuli while they were seated in a chair in a darkened room. The stimuli appeared on a $17^{\prime}$ monitor at a distance of approximately one meter from the participant.

We used the same images of complex natural scenes as stimuli as in (Lyyra et al., 2010) (see Figure 1). There were a total of 10 original images and their modified versions with different kinds of changes, whose presentation order was randomized across participants. Changes across the images referred to appearances or disappearances of objects, or changes in their position or color. The direction of the changes (disappearance vs. appearances of objects) was counterbalanced across participants. Each of the images was $500 \mathrm{~ms}$ in duration and blank screen, i.e., ISI, of $100 \mathrm{~ms}$ in duration in one condition, and $500 \mathrm{~ms}$ in another condition separated consecutive image pairs. Ten stimulus blocks with different 50 image pairs were presented. In five of the blocks, a $100 \mathrm{~ms}$ ISI was used, and $500 \mathrm{~ms}$ ISI in the other five blocks. The order of the blocks was counterbalanced across the participants.

An image with change was infrequently and pseudo-randomly interspersed ( $p=0.2$ ) with its frequently presented pair without change ( $p=0.8$ ) (oddball condition). There were up to seven but no less than three image presentations with no change separating image presentations with change.

The participants were instructed to search for a change in the images in each stimulus sequence, and to report of noticing the change for the first time by pressing a button. They were also instructed to silently count the number of the remaining observable change occurrences in the sequence. Change detection performance was measured for each subject as the average number of presentation cycles of the changed images in one stimulus sequence required for the explicit behavioral report of change detection (button press). Occasional button presses to misses and false alarms (less than 3\%) were used to distinguish them from change blindness, and successful detection was corrected on the basis of the counting task.

\section{EEG-RECORDINGS AND DATA-ANALYSIS}

Electroencephalography (EEG) was recorded with Brain Vision Recorder software (Brain Products GmbH, Munich, Germany) 


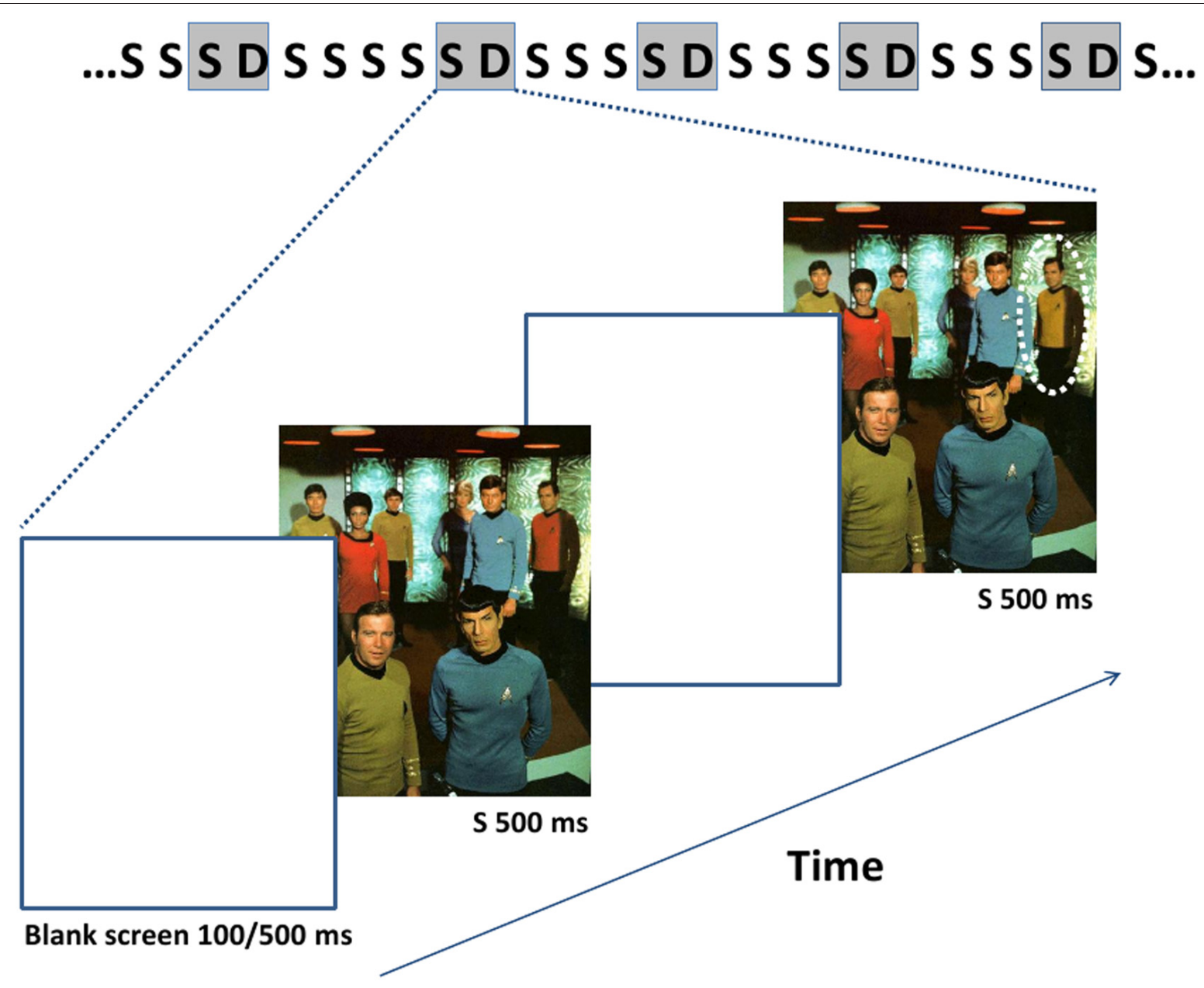

FIGURE 1 | The succession of the stimuli including an example of the images used is depicted uppermost with gray rectangles representing stimuli during which the EEG data were recorded. The probability of the appearance of the standard stimulus was 0.8 and that of the deviant stimulus
0.2. The duration of the standard (S) and deviant (D) images is $500 \mathrm{~ms}$ and that of the mask (ISI) either $100 \mathrm{~ms}$ or $500 \mathrm{~ms}$ throughout a stimulus block. The dotted circle indicates the site of the modification in the exemplar deviant image. from 29 channels of the international 10/20 system (FP1, FP2, Fz, F3, F4, F7, F8, FC1, FC2, FC5, FC6, Cz, C3, C4, CP1, CP2, CP5, CP6, T7, T8, Pz, P3, P4, P7, P8, Oz, O1, and O2) using an elastic cap (Electrocap) with $\mathrm{Ag} / \mathrm{AgCl}$ electrodes. Average reference was used in the recordings and the extraction of ERPs. An electrode on the forehead was used as a ground electrode. The original EEG signals were sampled continuously at $1000 \mathrm{~Hz}$, amplified and band-pass-filtered online with 0.1 to $100 \mathrm{~Hz}$. The data were further processed with Brain Vision Analyzer 2.0 (Brain Products GmbH, Munich, Germany). The independent component analysis (ICA) function of the Brain Vision Analyzer 2.0 software was used to identify and correct for blinks and eye movements in the EEG. Channels with excessive muscular artifacts were removed. Data with voltage gradient over $80 \mu \mathrm{V} / 200 \mathrm{~ms}$ and maximum and minimum voltages exceeding $\pm 80 \mu \mathrm{V}$ at any channel were marked as containing artifacts. The signals were off-line band-pass filtered from 0.1 to $30 \mathrm{~Hz}$. Sweeps containing artifacts were discarded for all channels, the average rejection rate being $12.4 \%$ (0 to $65 \%$, s.e. 20.89 ). Only subjects with more than 15 sweeps in the averaging after the artifact rejection were included in the ERP-averages (mean number of sweeps 57.7 for the $100 \mathrm{~ms}$ ISI condition and 74.1 for the $500 \mathrm{~ms}$ ISI condition). The rejection was done for each condition and all subjects, and this procedure lead to 18 subjects in each ISI condition (100 and $500 \mathrm{~ms}$ ) that were, however, not the same for both conditions.

As we were interested in implicit change processing, only data during change blindness, from the time period before the button press in the stimulus sequence were used for the ERP analysis. We excluded one presentation of change immediately preceding the button press not to confound implicit change processing with initial explicit change detection. For the ERP analyses, sweeps of the electrophysiological responses to the changed images and to the unchanged images immediately preceding them were picked in a time window of $100 \mathrm{~ms}$ before and $300 \mathrm{~ms}$ after stimulus onset. These responses were averaged 
across each subject. This way the sweeps were from responses to image pairs close to each other in the stimulus sequence, and a similar amount of responses to changed and original images included in the averages. A $100 \mathrm{~ms}$ pre-stimulus period served as a baseline, against which the ERPs were corrected in both conditions.

A prominent differential ERP for the unnoticed changes of negative polarity is observable in the grand averaged waveforms (see Figures 2 and 3 ) in the posterior and posterio-temporal electrode sites (P7, P8, Oz, O1, O2), especially in the condition of $100 \mathrm{~ms}$ ISI. These differential ERPs resembled in their latency, scalp topography, and stimulus and attentive conditions the visual analog of the mismatch negativity (MMN) of ERPs (Pazo-Alvarez et al., 2003; Czigler, 2007). For assessing response amplitudes, a time window of 200-260 ms (for vMMN, see e.g., Astikainen et al., 2008; Pazo-Alvarez et al., 2003; for change blindness studies, see Fernandez-Duque et al., 2003; Schankin and Wascher, 2007, 2008; Kimura et al., 2008) was selected for which mean values from each electrode were extracted. The resultant values were submitted to a repeated measures multivariate analysis of variance (MANOVA), Electrode site (P7, P8, Oz, O1, O2) and Stimulus type (No change, Change) as within-subject factors in each ISI condition $(100 \mathrm{~ms}$ and $500 \mathrm{~ms}$ ). This is due to two things. First, a response to the presentation of the blank screen is visible in the pre-stimulus period of the ERPs of the $100 \mathrm{~ms}$ ISI condition suppressing their amplitudes (see Figure 3), which makes the conditions differ slightly in functional terms with respect to each other. Second, the subjects are only partly same in both conditions, so they cannot be incorporated in the same statistical model. Only results involving Change as a factor are reported here.
Explicit change detection performance was measured by the average number of presentations of images containing a change in a stimulus sequence required for explicit report of initial change detection (button press). The performance in the distinct ISI-conditions was compared by paired $t$-tests.

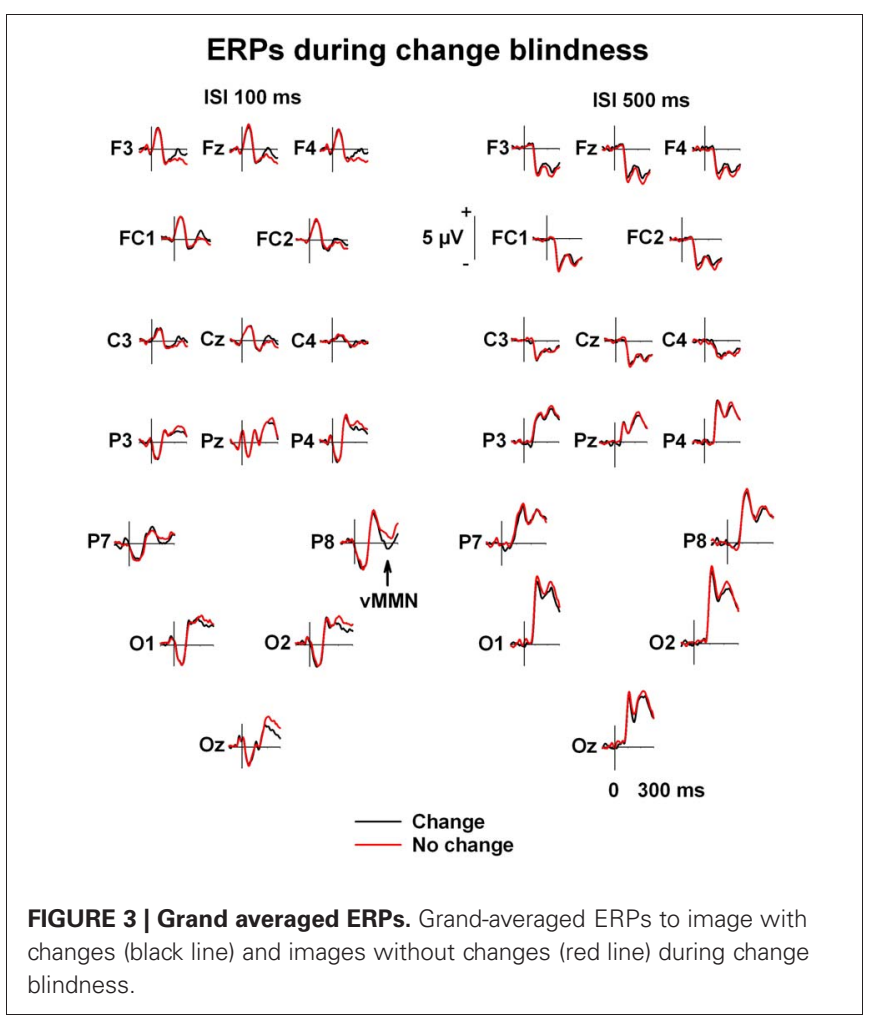

\section{Grand average difference waves (change - no-change)}

ISI $100 \mathrm{~ms}$

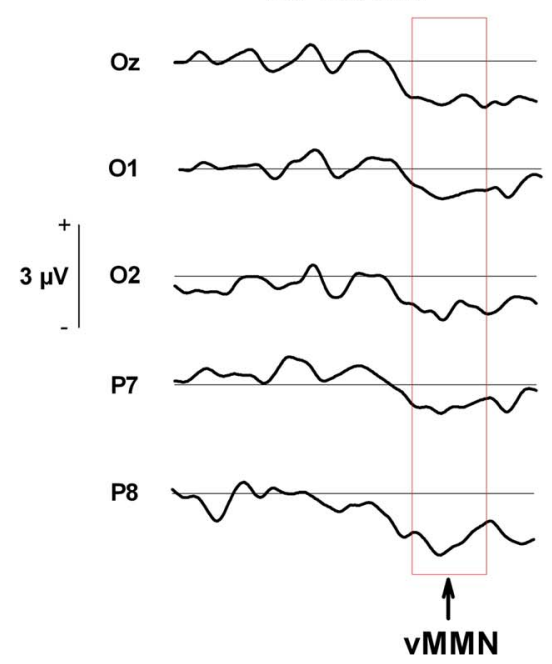

ISI $500 \mathrm{~ms}$

$\mathrm{Oz}$

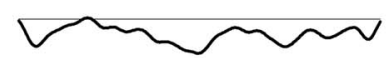

01

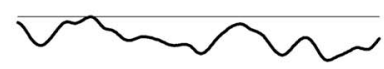

$\mathrm{O} 2$

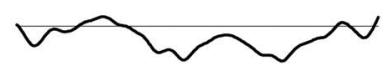

P7

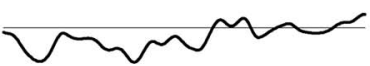

P8

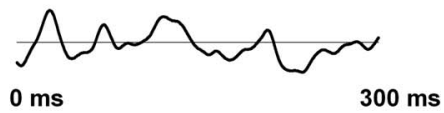

FIGURE 2 | Grand averaged difference waves (change-no change) during change blindness running from stimulus onset $(0 \mathrm{~ms})$ to $300 \mathrm{~ms}$ post-stimulus. 


\section{RESULTS \\ BEHAVIORAL DATA}

It took for the participants an average of 10.30 (s.e. 6.49) presentations of changed images in a stimulus sequence until they were able to detect the changes with the $100 \mathrm{~ms}$ ISIs while it took an average of 13.94 (s.e. 8.03) such presentations with $500 \mathrm{~ms}$ ISIs. This difference between these numbers was significant, $t(21)=2.15, p<0.05$, indicating a slightly earlier detection of the changes with the shorter ISIs.

\section{ELECTROPHYSIOLOGICAL DATA}

In the condition of $100 \mathrm{~ms}$ ISI, the MANOVA with Electrode site and Change as factors for the mean voltages in the time window of $200-260 \mathrm{~ms}$ revealed the main effect of Change, $[F(1,17)=8.06$, $p<0.02]$, indicating that changes were implicitly detected with the shorter ISI. The Electrode site $\times$ Change interaction was not significant, $[F(4,14)=1.45, p=0.27]$, indicating no specific role for any of the electrode sites in the detection (Figures $\mathbf{2}$ and $\mathbf{3}$ ).

In the stimulus condition of $500 \mathrm{~ms}$ ISI, the main effect of Change did not quite reach significance, $[F(1,17)=3.09, p=$ 0.097], nor did the interaction effect, $[F(4,14)=0.85, p=0.52]$.

\section{DISCUSSION}

We assessed explicit detection, as reflected by voluntary behavioral responses, and implicit detection, as reflected by ERPs of the brain, of a rare pair of images with change as interspersed with a repeated pair of images with no change. There was a blank interval of either $100 \mathrm{~ms}$ or $500 \mathrm{~ms}$ between the paired images across which the changes were produced. Both intervals allowed the explicit detection of the images with changes. The detection developed slightly faster across the trials with the $100 \mathrm{~ms}$ (after presentation of 11 changed images) than with the $500 \mathrm{~ms}$ ISI (after presentation of 14 changed images). Prior their explicit detectability, i.e., during change blindness, images with changes were detected implicitly, as reflected by the posterior electrical brain responses 200-260 ms after the change onset (vMMN) but only with the $100 \mathrm{~ms}$ ISI.

With the shorter ISI of $100 \mathrm{~ms}$, we found vMMN to unnoticed images with change. Similarly, traces of implicit change detection, as reflected by differential electrophysiological responses at 200-300 ms post-stimulus, have been observed by Schankin and Wascher in a couple of studies using a short ISI $(30 \mathrm{~ms})$ or transient change-occluding stimuli $(50 \mathrm{~ms}$ in Schankin and Wascher, 2007; $100 \mathrm{~ms}$ in Schankin and Wascher, 2008; cf. Fernandez-Duque et al., 2003; Kimura et al., 2008). More specifically, Schankin and Wascher observed a contralateral modulation of the N2pc-component at posterio-temporal electrode sites 220-260 ms (Schankin and Wascher, 2007), and 245-295 ms after stimulus onset (Schankin and Wascher, 2008) elicited by changes presented in the right or left visual hemifield, reflecting the location of the unnoticed change in the display.

Despite the longer ISI of $500 \mathrm{~ms}$, in comparison to $100 \mathrm{~ms}$ ISI, only slightly delayed the explicit detectability of images with changes, we failed to observe a robust vMMN to these images when $500 \mathrm{~ms}$ ISI was applied. This finding was expected since it is known that vMMN is dependent on active sensory memory trace of the standards (for the memory comparison hypothesis of
MMN, see Näätänen, 1992). Indeed, vMMN to even perceptually simpler changes (in orientation of a bar) in repeated visual stimuli has been found to disappear along with the lengthening of blank ISIs from $400 \mathrm{~ms}$ to $1100 \mathrm{~ms}$ (Astikainen et al., 2008). Previous studies with longer ISIs between the images delivering the change (900 ms in Henderson and Orbach, 2006; $500 \mathrm{~ms}$ in Eimer and Mazza, 2005) have failed to observe vMMN or other deflection in the N2-latency in response to unnoticed images with change. Indeed, Schankin and Wascher (2007) associated these negative findings with the extinction of the memory trace of the first image of a pair and, thereby, with the crash down of the whole process of comparison between the second and the first image of a pair. This account could well also explain our negative finding with the longer, $500 \mathrm{~ms}$ ISI and the positive one with the $100 \mathrm{~ms}$ ISI.

Our finding of the explicit detection of implicitly undetectable images with change questions the view that implicit change detection is needed for the development of the ability to detect changes explicitly. Furthermore, given the previous findings of implicit without explicit change detection (Fernandez-Duque et al., 2003; Eimer and Mazza, 2005; Schankin and Wascher, 2007, 2008; Kimura et al., 2008; Khittl et al., 2009; Lyyra et al., 2010), our finding of an opposite dissociation may even suggest that the implicit and explicit modes of change detection may recruit functionally independent neural mechanisms.

vMMN to images with change with the $100 \mathrm{~ms}$ intervals between the images of a pair may also have been modulated by other temporally overlapping ERP components linked to specific aspects of change, such as its target status or mere occurring probability (vMMN and N2pb, see Luck, Hillyard, 1994; Henderson and Orbach, 2006), its spatial location (N2pc, see Eimer and Mazza, 2005; Schankin and Wascher, 2007, 2008), or visual awareness of changes (visual awareness negativity, VAN, Koivisto and Revonsuo, 2003; Busch et al., 2010a,b). The N2pb has been associated with explicitly detected changes (Luck, Hillyard, 1994). Like N2pb, VAN has been linked to explicit change detection (Koivisto and Revonsuo, 2003) and also to "sensing" of presence of changes before their detection (Busch et al., 2010a,b). In the present study, a possibility of explicit detection of images with change was minimized. The threshold for the participants' responses to these images was set as low as possible. No identification of the changes was required and we excluded the last explicitly undetectable change in stimulus sequence from analyses and ERP-extraction, and it is for these change presentations that sensing or localization has been found to occur (Niedeggen et al., 2001; Mitroff et al., 2002). Accordingly, the N2pc component related to spatial attention has been observed in change blindness experiments specifically in the S1-S2 paradigm during change blindness (Schankin and Wascher, 2007, 2008) and "sensing" of the changes (Busch et al., 2010a,b). For these reasons, we consider that the differential ERP-response we observed for unnoticed changes was most likely vMMN in particular. Therefore, our study also leaves open the possibility that a shift of spatial attention to the changes is necessary, even if not sufficient, for explicit identification (see Watanabe, 2003; Schankin and Wascher, 2007, 2008; Busch et al., 2010a,b), and that vMMN reflects another type of implicit change detection that is not 
even necessary for explicit change detection. However, if it is unlikely that the vMMN we observed reflects localization of the changes or sensing of changes, it is not clear what kind of processing the vMMN we observed reflects in our study. Implicit change detection may only consist of registration of the features of the changing objects (Lyyra et al., 2010), although there is also interesting behavioral evidence suggesting that information about the identity of the changing objects or their features could be implicitly processed (Angelone et al., 2003). vMMN itself is a component that is elicited by single features but also for complexes of features (e.g., Müller et al., 2010). Moreover, it is even possible that vMMN does not reflect a unitary process throughout the period of change blindness but rather progressing (implicit) change detection (cf. Rensink, 2002). A study showing, e.g., distinct brain responses to implicit detection of random groups of single features versus organized combinations of the same features could shed more light on the issue of how far the changes are processed implicitly.

Some theorists have challenged the existence of implicit change detection (Mitroff et al., 2002). Their criticism lies in the reliability of behavioral measures of change awareness. Unconfidence of the subjects about the content of their perceptual experience and inconservative response criterions of explicit awareness may allow residual explicit awareness to affect indirect behavioral measures (see, however, Fernandez-Duque and Thornton, 2003; Laloyaux et al., 2006). In the present study, we used a fairly liberal response criterion, that is, button press at the first explicitly detectable occurrence of change not requiring identification. Nevertheless, even if we excluded the change occurrence immediately preceding explicit change detection from ERP-extraction, the subjects might have been marginally aware of or "sensed" the change even at some point before the button press. Therefore, the implicit nature of the detection stands in our study in the failure of the declarative consciousness to access the changes to allow the changes to be reported behaviorally. It is thereby possible that implicit changes may have made a difference in the phenomenal subjective experience of the subjects, even though they have not been able to access this information to report on it. Nevertheless, we think that our interpretation of the independence of the pre-attentive vMMN from explicit behavioral detection requiring focal attention holds.

Manipulating the duration of the blank interval led to a couple of incompatibilities with regard to the ERPs between the two ISI conditions. The amplitudes in the ISI 100 condition are decreased and their polarity even partly reversed due to the ERP-response to the blank interval (cf. Schankin and Wascher, 2007, 2008) as compared to the ISI500 condition in which the response to the blank screen has dissipated. We see this incompatibility as no threat to the present results, since the two ISI conditions were not compared to each other, only ERPs to changed and unchanged images within each ISI condition. For each ISI condition, the stimulus conditions are identical, and the differences can thus only be due to the unnoticed changes in the stimuli. Also because of surprisingly good change detection performance together with artifact rejection, the sweep numbers for ERP extraction were relatively low for some participants in either ISI condition. However, most sweep numbers were close to the average ( 57.7 for the $100 \mathrm{~ms}$ ISI condition and 74.1 for the $500 \mathrm{~ms}$ ISI condition), and we used methods such as ICA in ocular correction to enhance the signal to noise ratio. Moreover, the trial numbers were lower for the ISI100 condition than the ISI500 condition. Low numbers of trials rather occludes potential effects, but still the effect of change in ERPs reached significance in the ISI 100 condition, and the negative result in ISI500 condition was not due to its poorer signal to noise ratio. To further investigate the reliability of our results in this respect, we reanalyzed the results using only the data on the 12 participants with more than 30 trials in ERP-averages in both conditions. The results were similar to the original ones; the ERPs differed significantly in response to unnoticed changes in the ISI100 condition $(p<0.05)$, but not in the ISI500 condition $(p=0.09)$.

In the ERPs of the anterior electrode sites, there is a positive change-related amplitude difference visible at the same latency as the vMMN (see Figure 3). Positive differential ERPs have been reported accompanying vMMN in a number of studies (see Czigler, 2007 for a review). However, a similar ANOVA for the frontal electrode sites (F3, F4, Fz, FC1, FC2, Cz) as for the posterior electrode sites did not reveal any significant change-related amplitude differences.

The development of explicit change as a function of repeated occurrences of change without the guidance of implicit change detection would be in line with the view that a major factor in overcoming change blindness is top-down visual search strategy (Sampanes et al., 2008). In future studies, selectively affecting this strategy (attention to single objects as opposed to sets of objects) could provide a means to further explore on the aspects that dissociate explicit from implicit change detection, the latter of which rather utilizes elementary visual attributes. Nevertheless, we cannot exclude the possibility that some aspects of implicitly processed information about changes could affect their explicit detection. For example, it has been shown that socially relevant changes are detected faster than neutral ones (David et al., 2006) and that information about the emotional content of visual stimuli could be processed implicitly (Pasley et al., 2004). ERPs related to emotional processing could be used to investigate implicit processing of emotional contents of the changes, and their relation to change detection performance in the change blindness condition.

In sum, our results suggest that, despite implicit detection of visual changes remains unobservable, the changes still can build up an ability to detect the changes explicitly. Namely, despite the implicit detection of the visual changes fails due to long stimulation intervals beyond the temporal span of a memory system involved, the visual information, on which such detection is based, may still be successfully stored for these periods and used to prepare the visual system for the explicit detection of changes. Together with previous findings of implicit despite the absence of explicit change detection, our finding of the opposite dissociation suggests that implicit and explicit modes of change detection may recruit functionally distinct memory-based mechanisms of the brain.

\section{ACKNOWLEDGMENTS}

The authors wish to thank Lauri Viljanto for technical help. The study was supported by the Emil Aaltonen Foundation (project funding for Piia Astikainen). 


\section{REFERENCES}

Angelone, B. L., Levin, D. T., and Simons, D. J. (2003). The relationship between change detection and recognition of centrally attended objects in motion pictures. Perception 32, 947-962.

Astikainen, P., Lillstrang, E., and Ruusuvirta, T. (2008). Visual mismatch negativity for changes in orientation-a sensory memorydependent response. Eur. J. Neurosci. 28, 2319-2324.

Beck, D. M., Rees, G., Frith, C. D., and Lavie, N. (2001). Neural correlates of change detection and change blindness. Nat. Neurosci. 4, 645-650.

Busch, N. A., Fründ, I., and Herrmann, C. S. (2010a). Electrophysiological evidence for different types of change detection and change blindness. J. Cogn. Neurosci. 22, 1852-1869.

Busch, N. A., Dürschmid, S., and Herrmann, C. S. (2010b). ERP effects of change localization, change identification, and change blindness. Neuroreport 21, 371-375.

Chun, M. M., and Nakayama, K. (2000). On the functional role of implicit visual memory for the adaptive deployment of attention across views. Vis. Cogn. 7, 65-81.

Czigler, I. (2007). Visual mismatch negativity: violating of nonattended environmental regularities. J. Psychophysiol. 21, 224-230.

David, E., Laloyaux, C., Devue, C., and Cleeremans, A. (2006). Change blindness to gradual changes in facial expressions. Psychol. Belg. 46, 253-268.

Eimer, M., and Mazza, V. (2005). Electrophysiological correlates of change detection. Psychophysiology $42,328-342$.

Fernandez-Duque, D., Grossi, G., Thornton, I. M., and Neville, H. J. (2003). Representation of change: separate electrophysiological markers of attention, awareness, and implicit processing. J. Cogn. Neurosci. 15, 1-17.

Fernandez-Duque, D., and Thornton, I. M. (2000). Change detection without awareness: do explicit reports underestimate the representation of change in the visual system? Vis. Cogn. 7, 323-344.
Fernandez-Duque, D., and Thornton, I. M. (2003). Explicit mechanisms do not account for implicit localization and identification of change: an empirical reply to Mitroff et al. (2002). J. Exp. Psychol. Hum. Percept. Perform. 29, 846-858.

Goodale, M. A., and Milner, A. D. (1992). Separate visual pathways for perception and action. Trends Neurosci. 15, 20-25.

Hayhoe, M. M., Bensinger, D. G., and Ballard, D. H. (1998). Task constraints in visual working memory. Vision Res. 38, 125-137.

Henderson, R. M., and Orbach, H. S. (2006). Is there a mismatch negativity during change blindness? Neuroreport 17, 1011-1015.

Hollingworth, A., Schrock, G., and Henderson, J. M. (2001). Change detection in the flicker paradigm: the role of fixation position within the scene. Mem. Cognit. 29, 296-304.

Huettel, S. A., Güzeldere, G., and McCarthy, G. (2001). Dissociating the neural mechanisms of visual attention in change detection using functional MRI. J. Cogn. Neurosci. 13, 1006-1018.

Khittl, B., Bauer, H., and Walla, P. (2009). Change detection related to peripheral facial expression: an electroencephalography study. J. Neural Transm. 116, 67-70.

Kimura, M., Katayama, H., and Ohira, H. (2008). Event-related brain potential evidence for implicit change detection: a replication of Fernandez-Duque et al. (2003). Neurosci. Lett. 448, 236-239.

Kimura, M., Schröger, E., and Czigler, I. (2011). Visual mismatch negativity and its importance in visual cognitive sciences. Neuroreport 22, 669-673.

Koivisto, M., and Revonsuo, A. M. (2003). An ERP study of change detection, change blindness, and visual awareness. Psychophysiology 40, 423-429.

Laloyaux, C., Destrebecqz, A., and Cleeremans, A. (2006). Implicit change identification: a replication of Fernandez-Duque and Thornton (2003). J. Exp. Psychol. Hum. Percept. Perform. 32, 1366-1379.

Luck, S. J., and Hillyard, S. A. (1994). Spatial filtering during visual search: evidence from human electrophysiology. J. Exp. Psychol. Hum. 20, 1000-1014.

Lyyra, P., Wikgren, J., and Astikainen, P. (2010). Event-related potentials reveal rapid registration of features of infrequent changes during change blindness. Behav. Brain Funct. 6, 12.

Mack, A., and Rock, I. (1998). Inattentional Blindness. Cambridge, MA: MIT Press.

Mitroff, S. R., Simons, D. J., and Franconeri, S. L. (2002). The siren song of implicit change detection. J. Exp. Psychol. Hum. Percept. Perform. 28, 798-815.

Müller, D., Winkler, I., Roeber, U. Schaffer, S., Czigler, I., and Schröger, E. (2010). Visual object representations can be formed outside the focus of voluntary attention: evidence from event-related brain potentials. J. Cogn. Neurosci. 22, 1179-1188.

Näätänen, R. (1992). Attention and Brain Function. Hillsdale, NJ: Erlbaum.

Niedeggen, M., Wichmann, P., and Stoerig, P. (2001). Change blindness and time to consciousness. Eur. J. Neurosci. 14, 1719-1726.

O'Regan, J. K., and Noë, A. (2001). A sensorimotor account of vision and visual consciousness. Behav. Brain Sci. 24, 939-1031.

Pasley, B. N., Mayes, L. C., and Schultz, R. T. (2004). Subcortical discrimination of unperceived objects during binocular rivalry. Neuron 42, 163-172.

Pazo-Alvarez, P., Cadaveira, F., and Amenedo, E. (2003). MMN in the visual modality: a review. Biol. Psychol. 63, 199-236.

Pessoa, L., and Ungerleider, L. G. (2004). Neural correlates of change detection and change blindness in a working memory task. Cereb. Cortex 14, 511-520.

Rensink, R. A. (2000). Seeing, sensing, and scrutinizing. Vision Res. 40 1469-1487.

Rensink, R.A. (2002). Change detection. Annu. Rev. Psychol. 53, 245-277.

Rensink, R. A., O'Regan, J. K., and Clark, J. (1997). To see or not to see: the need for attention to perceive changes in scenes. Psychol. Sci. 8, 368-373.
Rensink, R. A., O’Regan, J. K., and Clark, J. (2000). On the failure to detect changes in scenes across brief interruptions. Vision Res. 7, 127-145.

Sampanes, A. C., Tseng, P., and Bridgeman, B. (2008). The role of gist in scene recognition. Vision Res. $48,2275-2283$.

Schankin, A., and Wascher, E. (2007). Electrophysiological correlates of stimulus processing in a change blindness paradigm. Exp. Brain Res. 183, 95-105.

Schankin, A., and Wascher, E. (2008). Unvoluntary attentional capture in change blindness. Psychophysiology 45, 742-750.

Simons, D. J., and Levin, D. T. (1997). Change blindness. Trends Cogn. Sci. $1,261-267$.

Sperling, G. (1960). The information available in brief visual presentations. Psychol. Monogr. 74, 1-29.

Watanabe, K. (2003). Differential effect of distractor timing on localizing versus identifying visual changes. Cognition 88, 243-257.

Yantis, S., and Jonides, J. (1984). Abrupt visual onsets and selective attention: evidence from visual search. J. Exp. Psych. Hum. Percept. Perform. 10, 601-621.

Conflict of Interest Statement: The authors declare that the research was conducted in the absence of any commercial or financial relationships that could be construed as a potential conflict of interest.

Received: 05 October 2011; accepted: 24 February 2012; published online: 13 March 2012.

Citation: Lyyra P, Wikgren J, Ruusuvirta $T$ and Astikainen $P$ (2012) Explicit behavioral detection of visual changes develops without their implicit neurophysiological detectability. Front. Hum. Neurosci. 6:48. doi: 10.3389/fnhum. 2012.00048

Copyright (c) 2012 Lyyra, Wikgren, Ruusuvirta and Astikainen. This is an open-access article distributed under the terms of the Creative Commons Attribution Non Commercial License, which permits non-commercial use, distribution, and reproduction in other forums, provided the original authors and source are credited. 